Rev. Elev. Méd, vét. Pays trop., 1978, 31 (4) : 443-445.

\title{
Note sur le sex ratio chez le zébu gobra au C.R.Z. de Dahra
}

\author{
par J. P. DENIS (*)
}

\begin{abstract}
RÉSUMÉ
Le sex ratio a été étudié sur plus de 3500 naissances de zébus Gobra entre 1954 et 1973. Il est de 0,50 en moyenne. Parmi les différents facteurs pouvant avoir une influence sur cette donnée (année de naissance, âge et influence propre de la mère, âge et infiuence propre du mâle), seule la saison semble avoir une influence. Il semble que les conditions défavorables puissent provoquer un plus grand nombre de naissances de femelles. L'importance pratique du fait ne serait cependant visible que dans le cas d'un regroupement des naissances à une période donnée de l'année. Dans tous les autres cas, l'équipartition des sexes reste la règle.
\end{abstract}

\section{INTRODUCTION}

La proportion de mâles et de femelles nés dans un troupeau est une notion importante à considérer puisqu'elle conditionne l'avenir de cet élevage sur le plan de la reproduction et de son évolution.

Disposant, au Centre de Recherches Zootechniques de Dahra-Djoloff au Sénégal, de données relatives aux naissances sur 20 années, il a paru intéressant d'analyser les chiffres obtenus.

En ce qui concerne le zébu Gobra, en dehors de cette station de recherches, rares sont les observations sur les troupeaux. Cependant, au cours des dernières années, deux enquêtes effectuées dans la région du Ferlo permettent d'avoir une idée de la répartition des sexes (tabl. 1).

(*) I. S. R. A., laboratoire national de l'Elevage et de Recherches vétérinaires, B. P. 2057, Dakar, Sénégal.
Il faut noter que ces enquêtes ont été effectuées pendant des années particulièrement rigoureuses au plan climatique (pluviométrie très diminuée par rapport à la moyenne) et, d'autre part, à la suite d'interrogatoires des éleveurs, ce qui peut introduire un biais important dans les résultats et expliquer, peut-être, le résultat exprimé par FAYOLLE.

\section{DONNÉES BIBLIOGRAPHIQUES}

La plupart des travaux effectués sur le sex ratio montrent des préoccupations essentiellement tournées vers la maîtrise de celui-ci. Un certain nombre cependant traite de la répartition des sexes en fonction de divers facteurs d'intervention possible. Ces facteurs sont les suivants :

- saison et année dont l'influence est reconnue ;

TABL. N*1-Répartition des sexes à la naissance chez le zébu Gobra.

(rêsultats d'enquêtes sur le terrain).

\begin{tabular}{|c|c|c|c|c|c|c|}
\hline \multirow{2}{*}{ Rëfêrence } & \multicolumn{2}{|c|}{ Nombre d'animaux } & \multicolumn{2}{|c|}{ en p. 100} & \multirow{2}{*}{$x^{2}$} & \multirow{2}{*}{ Signification } \\
\hline & Mâles & Femelles & Mâles & Feme11es & & \\
\hline $\begin{array}{l}\text { Fayolle et } \\
\text { Co1lab. } 1974\end{array}$ & 1607 & 2144 & 42,8 & 57,2 & 76,38 & HS \\
\hline Calvet 1973 & & & 45,3 & 53,7 & & \\
\hline
\end{tabular}


- état nutritionnel de la mère étroitement lié la plupart du temps au précédent ;

- âge des parents qui intervient assez nettement ;

— poids de la mère ;

- production laitière ;

- lignées maternelles à performances particulières dans ce domaine (bas pourcentage de produits d'un sexe donné);

- pH de la semence ;

- nutrition minérale des parents.

En fait, de très nombreux facteurs entrent en jeu. Leur nature et pleur importance sont très variables, ce qui pourrait expliquer les nombreuses divergences dans les conclusions. Ces différents facteurs vont être examinés chez le zébu Gobra.

\section{RÉSULTATS. — DISCUSSION}

\section{Valeur globale}

Elle est calculée sur les naissances survenues de 1954 à 1973 au nombre de 3507.

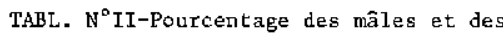
femelles chez le zểbu Gobra.

\begin{tabular}{|l|c|c|c|}
\hline \multicolumn{1}{|c|}{ Sexe } & Nombre & p.100 & $x^{2}$ \\
\hline Mâles & 1739 & 49,58 & \\
\cline { 1 - 3 } Femelles & 1768 & 51,42 & $\begin{array}{c}0,239 \\
\text { NS }\end{array}$ \\
\hline
\end{tabular}

Sur les 20 années d'observation il n'apparaît pas de différence significative dans la répartition des deux sexes.

\section{Sex ratio en fonction de l'année}

L'analyse de la régression de la proportion de mâles en fonction de l'année montre que la pente observée n'est pas significativement différente de $0(F=0,884$ NS à 0,05 p. 100$)$. On peut donc en conclure que le sex ratio ne montre pas de variations sensibles en fonction de l'année.

\section{Sex ratio en fonction de la saison}

Le tableau III montre l'évolution de la proportion de mâles en fonction de leur mois de conception en estimant la durée de la gestation à 10 mois ( $293 \pm 2$ jours).
Tableau $\mathrm{N}^{\bullet}$ III

\begin{tabular}{|c|c|c|c|c|c|}
\hline \multicolumn{2}{|c|}{ Mo.is } & $\begin{array}{l}\text { Nombre de } \\
\text { feme1les }\end{array}$ & $\begin{array}{c}\text { Nombre } \\
\text { de mâles }\end{array}$ & $\begin{array}{c}\text { p.100 } \\
\text { des mâles }\end{array}$ & $x^{2}$ \\
\hline \multicolumn{2}{|r|}{ Janvier } & 64 & 60 & $48,3-$ & 0,12 \\
\hline \multicolumn{2}{|r|}{ Février } & 83 & 54 & $39,4-$ & 6,13 \\
\hline \multicolumn{2}{|r|}{ Mars } & 104 & 73 & $41,2-$ & 5,42 \\
\hline \multicolumn{2}{|r|}{ Avril } & 120 & 129 & $51,8+$ & 0,32 \\
\hline \multicolumn{2}{|r|}{ Mai } & 123 & 121 & $49,5-$ & 0,01 \\
\hline \multicolumn{2}{|r|}{ Juin } & 108 & 98 & $47,5-$ & 0,48 \\
\hline \multirow{4}{*}{ 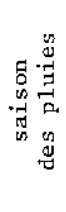 } & Juillet & 190 & 177 & $48,2 \sim$ & 10,46 \\
\hline & Août & 353 & 304 & $46,2-$ & 3,65 \\
\hline & Septembre & 298 & 321 & $51,8+$ & 0,85 \\
\hline & Octobre & 167 & 173 & $50,8+$ & 0,10 \\
\hline \multicolumn{2}{|r|}{ Novembre } & 104 & 135 & $56,4+$ & 4,02 \\
\hline \multicolumn{2}{|r|}{ Dé cembre } & 45 & 69 & $60,5+$ & 5,05 \\
\hline
\end{tabular}

Deux périodes peuvent être distinguées, une période qui va de septembre à décembre, durant laquelle on note une proportion plus importante de conceptions donnant naissance à des mâles, et une seconde période qui regroupe les autres mois de l'année à l'exception du mois d'avril. Sur le plan climatique, la première correspond à la période d'abondance, alors qu'ensuite s'installent des conditions nutritionnelles de plus en plus précaires. L'exception du mois d'avril confirme cette vue, puisque c'est à cette époque que se passe le phénomène de la montée de la sève. Les animaux privés de pâturage se tournent vers les jeunes pousses des arbres et complètent ainsi leur ration qui devient pourvue pendant quelques temps, en particulier sur le plan de matières protéiques. En fait, les différences ne sont significatives qu'entre les répartitions au cours des mois de février et mars d'une part, et novembre et décembre d'autre part.

\section{Sex ratio et apports minéraux}

Des enquêtes ont montré une relation nette entre dominantes minérales et répartition des sexes chez la vache (STOLKOWSKI). Pour cet auteur, un excédent de mâles se rencontre en présence d'un excès de potassium, et un excédent de femelles en cas d'excès d'alcalino-terreux. 
Enfin, dans le cas d'une équipartition des sexes, la valeur du rapport $\mathrm{K} / \mathrm{Ca}+\mathrm{Mg}$ est en moyenne de 1,5. Ceci a été vérifié au CRZ de Dahra (rapport $=1,49 \pm 0,05$, sex ratio 0,5 ) au cours de recherches systématiques sur l'évolution minérale sanguine au cours de l'année (CALVET).

\section{Autres facteurs}

Les autres facteurs considérés: âge de la mère, âge du père, influence du père ou de la mère, ne semblent pas avoir d'influences significatives sur les répartitions observées.

\section{CONCLUSION}

Le sex ratio a été étudié sur plus de 3500 naissances de zébus Gobra entre 1954 et 1973. Il est de 0,50 en moyenne. Parmi les différents facteurs pouvant avoir une influence sur cette donnée (année de naissance, âge et influence propre de la mère, âge et influence propre du mâle), seule la saison semble avoir une influence. Il semble que les conditions défavorables puissent provoquer un plus grand nombre de naissances de femelles. L'importance pratique du fait ne serait cependant visible que dans le cas d'un regroupement des naissances à une période donnée de l'année. Dans tous les autres cas, l'équipartition des sexes reste la règle.

\section{SUMMARY}

\section{Note on the sex ratio recorded in Gobra zebu cattle at the CRZ}

\section{of Dahra}

The sex ratio has been recorded for more than 3500 births of Gobra zebus between 1954 and 1973. It averages 0.50 . Among the various factors which can influence these data (Year of birth, mother's age and own influence, father's age and own influence), only the season seems to have a real influence. Unfavourable conditions seem to initiate a larger number of female births. However, the practical importance of this fact would be visible only if births were gathered at a given time of the year. In all the other cases, the sex equidistribution remains the rule.

\section{RESUMEN}

\section{Nota sobre el sex-ratio en el cebú Gobra en el Centro de Investigaciones zootécnicas de Dahra (Senegal) |}

Se estudió el sex-ratio en más de 3500 nacimientos de cebues Gobra entre 1954 y 1973 . Llega a 0,50 por término medio. Entre los diferentes factores pudiendo tener una influencia sobre este dato (año del nacimiento, edad e influencia propia de la madre, edad e influencia propia del macho), sóla la estación parece tener una influencia. A lo que parece, las condiciones desfavorables pueden provocar un mayor número de nacimientos de hembras. La importancia práctica de esto no seria sin embargo visible más que en el caso de un reagrupamiento de los nacimientos durante un periodo determinado del año. En todos los otros casos, la repartición igual de los sexos es requisito indispensable.

\section{BIBLIOGRAPHE}

L'essentiel des renseignements bibliographiques utilisés dans ce travail est tiré de « Annotated bibliography $n^{0} 186$ », préparé à partir de « Animal breeding abstracts » (1949-1971). Commonwealth bureau animal breeding and genetics.

1. BRANDS (A. F. A.), BANERJEE-SCHOTSMAN (I.), VAN DIETEN (S. W. J.), VAN LOEN (A.). Sex ratio of calves at birth. Anim. Breed. Abstr., 1965, $n^{\circ} 3331$.

2. CALVET (H.). Rapport concernant les premiers résultats obtenus au Centre de prévulgarisation de Labgar. Dakar, Lab. Nat. Elev. Rech. vet, avrıl 1973, $788 \mathrm{p}$.

3. FAYOLle (A. F.), COSTIOU (P.), GRANGE (M.). Valorisation du cheptel bovin. Zone sylvo-pastorale de la République du Sénégal. Rapport d'enquêtes, mars 1974, 126 p. (Convention FAC no 63/C/70/A. Projet $178 / \mathrm{CD} / 70 / \mathrm{VI} / \mathrm{A} / 5)$.
4. FRIOT (D.), CALVET (H.). Service de physiologienutrition. Rapport annuel. Dakar, Lab. Nat. Elev. Rech. Vét., 1970.

5. GUSE (H. O.). The effect of the $\mathrm{pH}$ value of semen and of cervical secretion on fertility and sex ratio in conditions unfavorable to fertility (studied in cattle and sheep). Kühn. Arch., 1957, $71: 211-255$.

6. KAMALJAN (V. S.). The effect of age of parents on the sex ratio of the progeny. Anim. Breed. Abstr., $1962, \mathrm{n}^{0} 793$.

7. SINGH (B.), SINGH (B. P.). The bearing of season and sequence of calving on frequency of male, female and total calvings in Hariana cows. Indian vet. J., $1968,45: 852-858$.

8. STOLKOWSKI (J.). Influence possible de la nutrition minérale sur la répartition des sexes chez la vache : une enquête rétrospective. $C . R$. hebd. Séanc. Acad. Sci., Ser. D., Paris, 1967, 265 : 1059-1062. 